\title{
Juvenile Aggressive Cemento-ossifying Fibroma of the Maxilla
}

\author{
Sampan Singh Bist, Saurabh Varshney, Sanjeev Bhagat, Sarita Mishra, Vinish Aggarwal
}

\begin{abstract}
Fibro-osseous lesions of the face and paranasal sinuses are relatively uncommon. The biological characteristics of fibroosseous lesions range from indolent to aggressive and from inflammatory to neoplastic. Cemento-ossifying fibroma is a rare, benign fibro-osseous lesion composed of lamellar bone, fibrous tissue and cementum. It is usually found in the jaw bone and presents in several variant pathological subtypes. The overlapping clinical, radiologic and histomorphologic features of these subtypes have led to diagnostic dilemma for the clinician, radiologist and the histopathologist. Clinically, the lesions are usually asymptomatic, slow-growing and wellcircumscribed. However, in very few cases, these tumors have demonstrated an aggressive course of development. Lesions that involve the midface and paranasal sinuses and presenting in earlier age tend to demonstrate aggressive behavior and rapid growth. We describe a case of a 13 years old young boy with aggressively behaving cemento-ossifying fibroma of the maxilla presenting acutely as right cheek swelling with gingival growth.
\end{abstract}

Keywords: Fibro-osseous lesions, Cemento-ossifying fibroma, Maxilla.

How to cite this article: BistSS, Varshney S, Bhagat S, Mishra $\mathrm{S}$, Aggarwal V. J uvenile Aggressive Cemento-ossifying Fibroma of the Maxilla. Int J Otorhinolaryngol C lin 2012;4(3):156-159.

\section{Source of support $\mathrm{Nil}$}

\section{Conflict of interest: None declared}

\section{INTRODUCTION}

In recent years, the term fibro-osseous lesions has grow $n$ in popularity as an overall designation for a number of rare, histologically benign lesions of the head and neck that are made up of bone, fibrous tissue and cementum. ${ }^{1}$ Presence of cementum or bone classifies the lesion as cementifying fibroma or ossifying fibroma respectively, while lesions with mixture of both cementum and bone matrix are known as cemento-ossifying fibroma (COF). A ccording to the World Heal th Organization (WHO) classification in 1992, COF is classified within nonodontogenic lineage tumors. ${ }^{2}$ The mesodermal origin of the tumor has been widely accepted, and it is thought to be originating from the periodontal ligament. ${ }^{3}$ This is a layer of fibrous connective tissue surrounding the roots. It contains multipotential cells capable of forming cementum, lamellar bone and fibrous tissue. Under pathological conditions, neoplasms containing any or all of the components may be produced. ${ }^{4} \mathrm{COF}$ is a locally destructive, deforming tumor that can occur almost anywhere in the craniofacial region. While these lesions are commonly seen in bone, their existence has been reported in soft tissue as well. M ost osseous lesions have been reported in the premolar-molar region of the mandible; other sites include the maxilla, zygoma, paranasal sinuses, orbital and petromastoid regions. ${ }^{5,6} \mathrm{M}$ ost extraosseus lesions have been reported in the gingiva. ${ }^{3}$ Trauma is widely accepted as the predisposing factor for these lesions. ${ }^{3,5,6}$ Exodontia and periodontitis have been shown to induce jaw lesions. ${ }^{3}$ COF occurs in patients of a wide age range, but most cases are encountered during the third and fourth decades of life and more commonly in women. ${ }^{5}$ They manifest themselves as asymptomatic, slow growing and well circumscribed intraosseous masses. These lesions, which can be either unilocular or multilocular, may give rise to growth of the jaw, leading to considerable esthetic and functional deformities. The clinical behavior and radiological appearance of fibro-osseous lesions is variable, based on evolution time and development phase. Understanding the nature of fibro-osseous lesion facilitates appropriate management. A ggressive lesions require a radical-surgical approach to ensure complete excision, despite an increase in associated morbidity. Incomplete excision of aggressive lesions may result in recurrence with severe morbidity or mortal ity. In contrast, a slow progressive lesion often does not warrant extensive surgical excision. Here, we present a case of aggressive COF of the maxilla in a young boy in whom the lesion recurs after curettage and enucleation as a primary surgical procedure.

\section{CASE REPORT}

A 13-year-old boy was referred by a dental surgeon to the Department of Otorhinolaryngology for the management of recurrent COF in the right upper jaw. The patient had visited the dental surgeon 1 year ago for a swelling in the upper premolar-molar region. At that time, the dental surgeon had performed surgical excision of the swelling and the histopathology of the excised specimen had revealed the diagnosis of COF. The patient had become asymptomatic after that but, in due course of time, he developed right cheek swelling along with swelling over the gingiva since last 4 months. The patient was then referred to our hospital for further management. External examination of the face revealed a smooth, bony hard and nontender mass in the right anterior maxillary region. On intraoral examination 


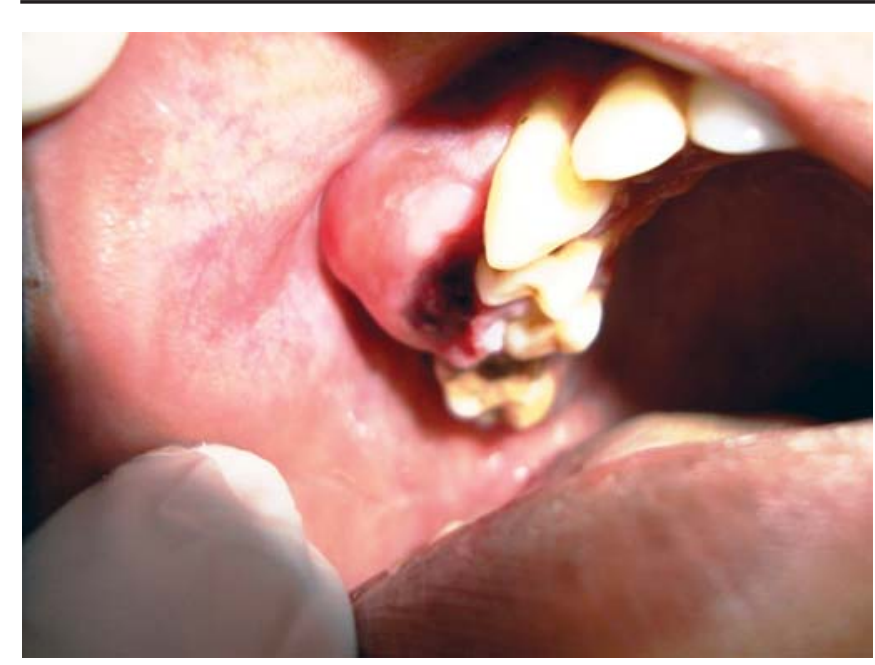

Fig. 1: Clinical intraoral photograph showing the lesion

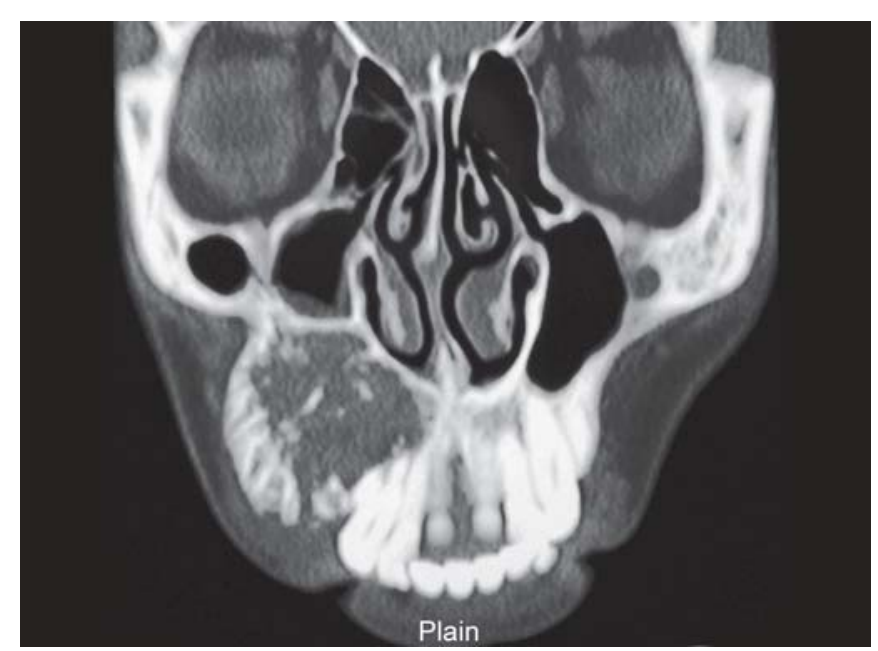

Fig. 2: Coronal CT scan showing the mixed radiolucentradiopaque lesion

(Fig. 1), a bony hard swelling in upper gingiva was seen which had obliterated the gingivobuccal sulcus and extended from canine to second molar. The adjacent teeth were loose and displaced. Remaining head and neck and systemic examination was unremarkable. Computed tomographic (CT) scan (Fig. 2) showed a mixed density mass with diffuse scattered calcification involving the right maxillary alveolar ridge, occupying and expanding the maxillary sinus. The mass was well circumscribed and showed both radiolucent and radiopaque features. A $n$ intraoral biopsy was performed to confirm the type of lesion and the results of histopathological examination were consistent with aggressive COF. The patient was managed by surgical resection via an extended sublabial approach (Fig. 3) and tumor was removed in piece meal. The $0^{\circ}$ endoscope was used for facilitating the complete removal of the tumor from the maxillary sinus. The excised surgical specimen reconfirmed the diagnosis of COF. The postoperative period was uneventful. There is no clinical sign of recurrence on

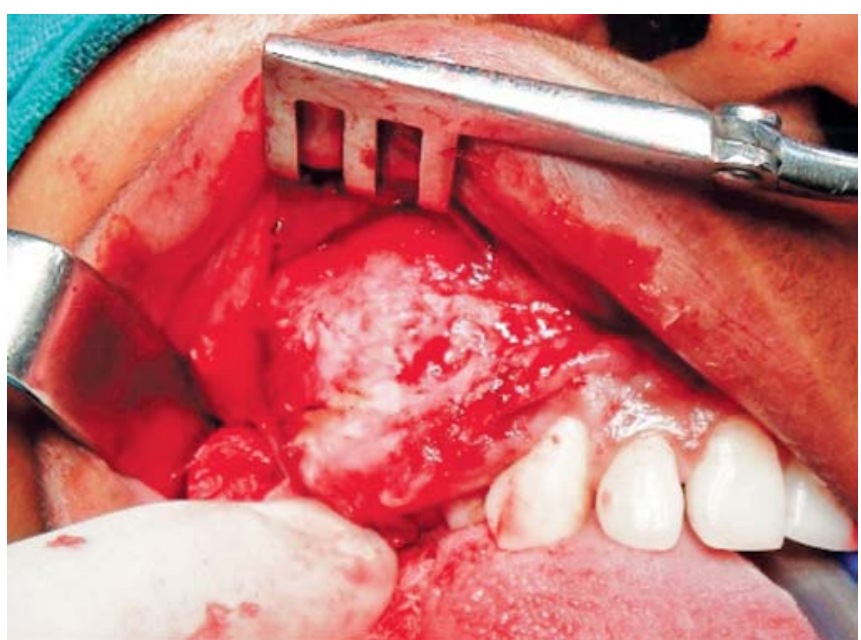

Fig. 3: Intraoperative photograph showing the bony lesion

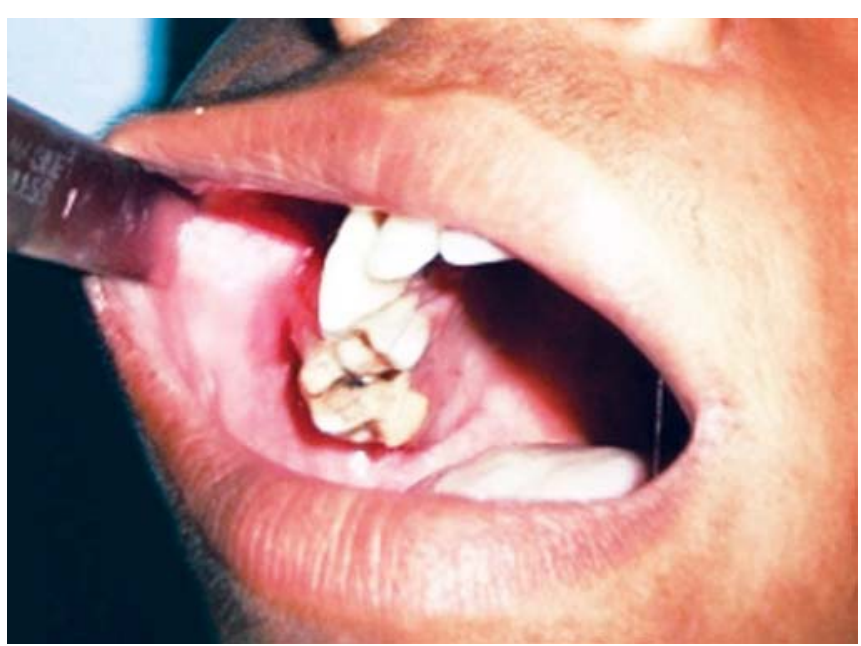

Fig. 4: Clinical intraoral photograph on follow-up

intraoral examination (Fig. 4) at 1 year and the patient is advised regular follow-up.

\section{DISCUSSION}

The WHO lists four kinds of fibro-osseous lesions: COF, fibrous dysplasia, ossifying fibroma and cementifying fibroma. ${ }^{2} \mathrm{COF}$ tends to be asymptomatic in the initial phases and is frequently diagnosed in advanced stages. There is localized increase in volume which is usually painless and leads to considerable esthetic and functional deformity. Maxillary COF are large at the time of presentation, indicating the capacity of the tumor to expand freely within the maxillary sinus. When this tumor arises in children, it is called the juvenile aggressive COF, which presents at an earlier age and is more aggressive clinically and more vascular at pathologic examination. ${ }^{7}$ Our patient was a young boy presenting with a giant lesion, which was involving the maxillary sinus and was aggressive in nature showing recurrence within few months after primary 
surgery. Histopathologically, COF shows a proliferation of irregularly shaped calcifications within a hypercellular fibrous connective tissue stroma. The calcifications are extremely variable in appearance and represent various stages of bone and cementum deposition. The histopathology of our patient had a cellular fibrous stroma with rounded masses of cement al ong with hypervascularity, which was suggestive of aggressive COF. Radiologically, COF appears as a well-circumscribed, solitary radiolucency with scattered radiopaque foci. It shows distinct stages during its development. In the early stages, the COF appears as a radiolucent lesion with no evidence of internal radiopacities. As the tumor matures, there is increasing calcification so that the radiolucent area becomes flecked with opacities until ultimately the lesion appears as an extremely radiopaque mass. The lesion in our patient had mixed density mass with diffuse scattered radiopacities. One additional important diagnostic feature is that there is a centrifugal growth pattern rather than a linear one and therefore the lesion grows by expansion equally in all directions and expands the surrounding cortical bone without perforation. They maintain a spherical shape and displacement of adjacent teeth is common. Surgery is the mainstay of treatment for COF. It usually shells out easily at surgery, but maxillary lesions are more difficult to remove completely than mandibular. This may be attributable to the difference in bone character between the mandible and maxilla and to the available space for expansion in the maxillary sinus. Treatment of aggressive lesion requires radical surgical approach. Growth of the facial skeleton is an important consideration in the pediatric age group. ${ }^{8}$ Extensive tumors in this age group are more difficult to manage since the tumors here tend to be more invasive, aggressive and have more chances of recurrence. $M$ andibular tumors can be treated with conservative surgery but aggressive surgery is warranted for midface and paranasal sinuses because of their more aggressive behavior. ${ }^{9}$ Even though small tumors can be removed by simple curettage and enucleation, they are better avoided for the fear of recurrence especially in juvenile COF. Our patient was also managed initially by simple curettage and enucleation and therefore the recurrence was seen probably due to incomplete removal. One study showed a recurrence rate of $28 \%$ after curettage. ${ }^{10}$ Endoscopic removal has al so been tried successfully for these tumors. ${ }^{11}$ But these conservative techniques make histological interpretation more difficult, especially in cases of hybrid lesions. Wherever possible, an open surgical technique is advised for adequate visualization and complete excision of these tumors. ${ }^{12}$ The extended Caldwell-Luc approach and sublabial approach are more suitable for lesions involving the maxilla and the premaxilla and have the advantage of having no external scar. We preferred the extended sublabial approach in our patient as it was a revision surgery for recurrence and the lesion was of large size. Lateral rhinotomy approach is suitable for the lesions involving the sinonasal cavity. Endoscopic assisted excision can be used with other approaches to facilitate the complete removal of the tumor especially, when the lesion is extensive and has to be removed piecemeal. We also used the $0^{\circ}$ endoscope to ensure the complete removal of tumor from maxillary sinus in our patient. Recurrence has been reported in as many as $28 \%$ of patients with mandibular COF. The recurrence rate of maxillary COF is unknown, but it is likely to be higher because of the greater difficulty of their surgical removal and larger size at the time of presentation. ${ }^{7}$ Our patient also reported recurrence after primary excision of the tumor involving maxillary sinus. The overall prognosis of COF appears to be good. Despite their tendency for local invasion and recurrence, there are no reported instances of metastatic disease. M alignant transformation is very rare. ${ }^{9}$

\section{CONCLUSION}

The COF that arises in the childhood in the maxillary region behaves aggressively and spreads rapidly despite repeated surgery. Hence, early radical surgical intervention is often necessary to control recurrence.

\section{REFERENCES}

1. Blayney A W, el Tayeb AA. The 'hybrid' fibro-osseous lesion. J Laryngol Otol 1986;100(3):291-302.

2. K ramer IRH, Pindborg JJ, Shear M. N eoplasms and other lesions related to bone. In: World Health Organization International Histological Classification of Tumors. New Y ork: SpringerVerlag 1992:28-31.

3. M artin Granizo R, Sanchez-Cuellar La, Falahat F. Cementoossifying fibroma of the upper gingivae. O tolaryngol Head N eck Surg 2000;122:755.

4. M agu S, A iron RK, M ishra DS, Y adav RK, K akkar V. Images: Cemento-ossifying fibroma of the maxilla. Ind J Radiol Imag 2000;10(2):103-04.

5. B rademann $G$, Werner JA, Janig $U$, et al. Cemento-ossifying fibroma of the petromastoid region: Case report and review of the literature. J Laryngol Otol 1997;111:152-55.

6. Waldron CA. Fibro-osseous lesions of the Jaws. J Oral M axillofac Surg 1993;51:828-35.

7. Kuta AJ, W orley CM , K augars GE. Central Cemento-ossifying fibroma of the maxillary sinus: A review of six cases. AJNR A m J Neuroradiol 1995;16:1282-86.

8. B hat KV, Naseeruddin K. Sublabial approach to sinonasal juvenile ossifying fibroma. Int J Pediatr Otorhinolaryngol 2002;64:239-242.

9. Commins DJ, Tolley NS, M ilford CA. Fibrous dysplasia and ossifying fibroma of the paranasal sinuses. J Laryngol Otol 1998;112(10):964-68. 
10. Eversole LR, Leider AS, Nelson K. Ossifying fibroma: A clinicopathologic study of 64 cases. Oral Surg Oral Med Oral Pathol 1985;60(5):505-11.

11. Choi $Y C$, J eon EJ, Park Y S. Ossifying fibroma arising in the right ethmoid sinus and nasal cavity. Int J Pediatr Otorhinolaryngol 2000;54:159-62.

12. Wenig BM , V inh TN, Smirniotopoulos J G, Fowler CB, H ouston GD, H effner DK . A ggressive psammomatoid ossifying fibromas of the sinonasal region: A clinicopathologic study of a distinct group of fibro-osseous lesions. Cancer 1995;76(7):1155-65.

\section{ABOUT THE AUTHORS}

\section{Sampan Singh Bist (Corresponding Author)}

A ssociate Professor, D epartment of ENT, Himalayan Institute of M edical Sciences, Dehradun, Uttarakhand, India, e-mail: sampanbist@ yahoo.com

\section{Saurabh Varshney}

Professor, Department of ENT, Himalayan Institute of Medical Sciences, Dehradun, Uttarakhand, India

\section{Sanjeev Bhagat}

A ssistant Professor, Department of ENT, Himalayan Institute of Medical Sciences, D ehradun, Uttarakhand, India

\section{Sarita Mishra}

A ssistant Professor, Department of ENT, Himalayan Institute of M edical Sciences, Dehradun, Uttarakhand, India

\section{Vinish Aggarwal}

Senior R esident, Department of ENT, Himalayan Institute of M edical Sciences, Dehradun, Uttarakhand, India 